\title{
Mechano-Optical Behavior of Loosely Crosslinked Double-Network Hydrogels: Modeling and Real-Time Birefringence Measurement during Uniaxial Extension
}

\author{
S. Shams Es-haghi, ${ }^{1,2,3}$ I. Offenbach, ${ }^{1 \dagger}$ D. Debnath, ${ }^{1}$ R. A. Weiss, ${ }^{1}$ M. Cakmak ${ }^{1,2,3,4^{*}}$ \\ ${ }^{1}$ Department of Polymer Engineering, The University of Akron, \\ Akron, Ohio, 44325-0301, USA \\ ${ }^{2}$ Birck Nanotechnology Center, Purdue University, \\ West Lafayette, Indiana 47907-2057, USA \\ ${ }^{3}$ School of Materials Engineering, Purdue University, \\ West Lafayette, Indiana 47907-2045, USA \\ ${ }^{4}$ School of Mechanical Engineering, Purdue University, \\ West Lafayette, Indiana 47907-2088, USA
}

\begin{abstract}
Real-time uniaxial strain-induced birefringence in loosely crosslinked double-network (DN) hydrogels synthesized from acrylamide (AAm) and $N, N$-dimethyl(acrylamide) (DMA) was measured. Tensile tests were performed at different extension rates from quasi-static conditions to very rapid tests followed by a holding for relaxation. Both DN hydrogels exhibit negative birefringence whose absolute value increased with extension and remained constant during relaxation. DN hydrogel synthesized from AAm displayed a linear stress optical (birefringence true stress) behavior, however, a nonlinear trend was observed for the DN gel synthesized from DMA. A simple photoelastic model was developed based on the mechanical behavior of the gels using Fung elastic potential and Brewster's stress-optical law, and the model was compared with the experimental data.
\end{abstract}

*Corresponding author: cakmak@purdue.edu

${ }^{\dagger}$ Current address: Evonik Corporation, Nutrition care Division, Richmond, Virginia 23237, USA 


\section{INTRODUCTION}

Hydrogels are hydrophilic polymeric networks that absorb large amounts of water, but the network structure that is formed by chemical or physical crosslinks is insoluble [1]. Hydrogels are similar to biological tissues and this makes them attractive candidates for various applications in bioengineering [2]. The optical properties of hydrogels become important when they are used in ophthalmology, especially for contact lenses [3]. For example, with a birefringent hydrogel, it is possible to make multifocal contact lenses [4,5]. Apart from practical purposes, the measurement of optical properties such as birefringence can provide a deeper understanding of the orientation of polymer chains and development of optical anisotropy within a material during deformation. The first study of strain-induced birefringence of hydrogels was reported by Hrouz and Ilavský [6] who measured the birefringence of a loosely crosslinked polyacrylamide gel in a mixture of acetone and water for very small elongation, less than $20 \%$. Geissler et al. [7] reported birefringence development during polymerization of polyacrylamide gels, which they termed spontaneous birefringence. They concluded that the birefringence was the result of the development of strain during the gelation process.

In order to study strain-induced birefringence in hydrogels under finite deformations, the hydrogel must be strong enough to be stretched to high stretch ratios. Conventional chemically crosslinked hydrogels are usually mechanically weak and break at relatively low extensions. Although there are various strategies to synthesize tough hydrogels [8], few studies considered or measured strain-induced birefringence of hydrogels in finite tensile deformation. Murata and Haraguchi [9] measured birefringence in nanocomposite hydrogels synthesized from $N$-isopropyl acrylamide under finite deformations. They observed a reversion in birefringence data of nanocomposite hydrogels in uniaxial deformation. Wu et al. [10] synthesized a robust optically 
anisotropic hydrogel by combining an anisotropic, physically cross-linked network of semirigid poly(2,2'-disulfonyl-4,4'-benzidine terephthalamide) (PBDT) and a chemically cross-linked network of polyacrylamide (PAAm) based on the double-network concept developed by Gong et al. [11]. They measured the birefringence of PBDT/PAAm gels by a polarizing optical microscope after elongating the hydrogel samples to a certain strain using a vernier caliper, and observed reversion in the birefringence data. Recently, Shikinaka et al. [12] measured birefringence in extended hydrogels synthesized from acrylamide and imogolite nanotubes. They found that by increasing the concentration of nanotubes a reversion is observed in birefringence data. In all those investigations, however, the birefringence was measured off-line, after the hydrogel was deformed, which raises the question of the effects of polymer relaxation during the time between the stretching and the birefringent measurement. Real-time measurements eliminate that problem and provide a clearer understanding of the variation of birefringence during deformation. In situ mechano-optical measurements allow one to directly measure the birefringent behavior over the entire deformation history, in contrast to off-line measurements that can realistically access only discrete strains. Similarly, the measurement of birefringence at very low stretch ratio and low stress, which is of crucial importance for calculating the stressoptical coefficient - especially for non-linear deformation, is a daunting challenge for off-line measurements, but easily achievable with real-time experiments. In effect, since in off-line measurements there is no way to measure the load applied on the sample, it is not possible to see the variation of birefringence as a function of stress which is crucial in measuring the stressoptical coefficient.

This paper reports the first real-time birefringence measurements of chemically crosslinked hydrogels under finite tensile deformation. Specifically, the development of strain- 
induced birefringence is reported for two double-network (DN) hydrogels, one synthesized from acrylamide (AAm) and the other from $N, N$-dimethyl(acrylamide) (DMA). In order to perform birefringence measurements during finite deformations, the hydrogel must be tough enough to sustain the load. Recently, Shams Es-haghi and Weiss [13] developed a method to fabricate tough chemically crosslinked hydrogels from multiple neutral networks. This method will be used to make tough DN hydrogels from AAm and DMA. The stress-optical coefficient is independent of network topology, such as crosslink density, but it is sensitive to the chemical structure of the network, which affects the mean refractive index of the material [14]. Thus, with the two DN hydrogels that were used in this research, it was possible to determine the effect of the chemical structure on the development of strain-induced birefringence.

\section{EXPERIMENTAL SECTION}

Materials. Acrylamide (AAm) and N,N'-methylenebis(acrylamide) (MBAA) were purchased from Sigma-Aldrich Chemical Co. and used as received. $N, N$-dimethyl(acrylamide) (DMA) was obtained from Sigma-Aldrich Chemical Co. and was vacuum distilled prior to use. A photoinitiator, 2-oxoglutaric acid (OXGA), was obtained from Fluka Chemical Co. and used as received.

Synthesis of Double Networks. DN hydrogels were synthesized by a two-step sequential free-radical polymerization [13]. The first network was prepared by adding OXGA and MBAA to a $4 \mathrm{M}$ solution of AAm in deionized (DI) water. The monomer solution was injected into a glass mold made of two parallel glass slides, which was then exposed for 20 min to $365 \mathrm{~nm}$ ultraviolet (UV) light $\left(15 \mathrm{~mW} / \mathrm{cm}^{2}\right)$, see Scheme 1. The resulting single-network (SN) hydrogel was then immersed into the same reaction mixture until an equilibrium swelling was 
achieved. The AAm-swollen SN gel was then placed between two parallel glass slides and exposed for $20 \mathrm{~min}$ to $365 \mathrm{~nm}$ ultraviolet (UV) light $\left(15 \mathrm{~mW} / \mathrm{cm}^{2}\right)$ to produce a AAm-DN hydrogel, Scheme 1. DMA-DN hydrogels were synthesized with the same procedure, Scheme 2.

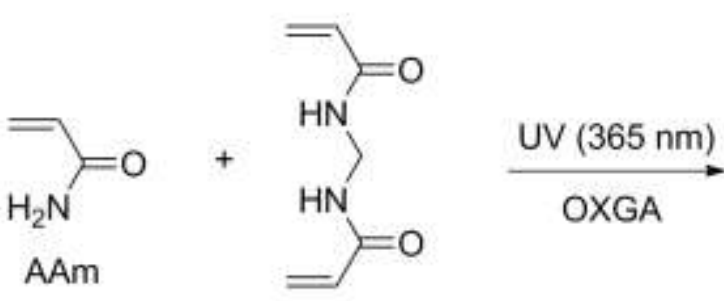

MBAA

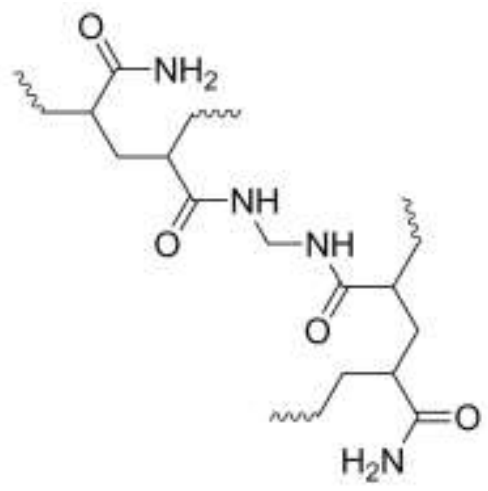

Scheme 1. Synthesis of poly(acrylamide) network.

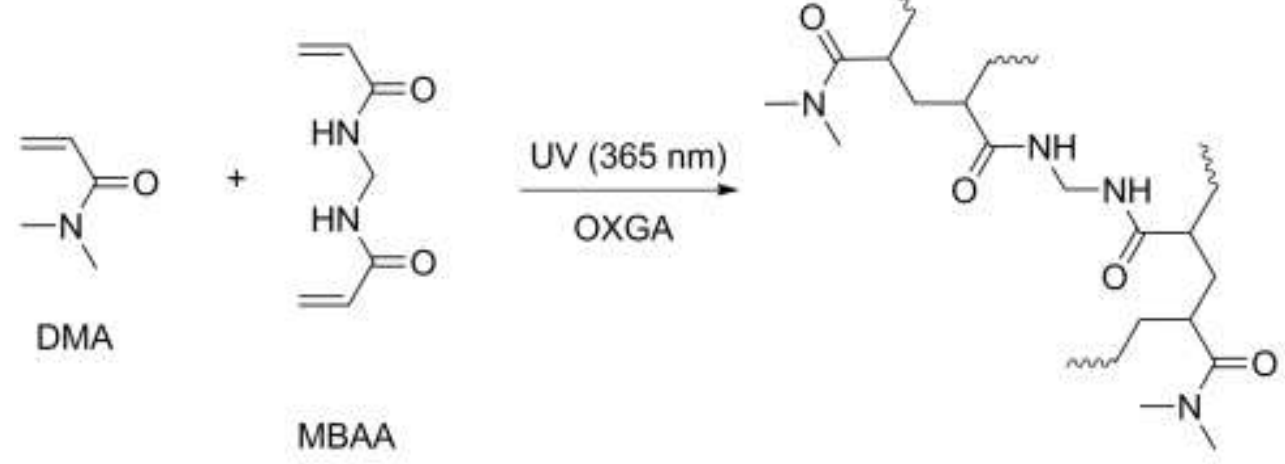

Scheme 2. Synthesis of poly( $N, N$-dimethyl(acrylamide) $)$ network.

Any unreacted monomer was removed by immersing the DN hydrogels in DI water, which was replaced a number of times with fresh water. Each individual network is described by the name 
of the monomer used, followed by four numbers in parenthesis that indicate 1) the molar concentration of monomer in DI water, 2) the mol\% of photoinitiator with respect to monomer, 3) the mol\% of crosslinking agent with respect to monomer and 4) UV dose used for the reaction (i.e., intensity $\times$ time of exposure). Thus, $\operatorname{DMA}(4,0.1,0.01,18)$ corresponds to the polymerization of a 4 M DMA solution using $0.1 \mathrm{~mol} \%$ OXGA, $0.01 \mathrm{~mol} \%$ MBAA and a UV dose of $18 \mathrm{~J} / \mathrm{cm}^{2}$. Table 1 summarizes the recipes used to synthesize the DN hydrogels in this study.

Table 1. Recipes for the DN Hydrogels

\begin{tabular}{cc}
\hline Recipe & DN Hydrogel Notation \\
\hline AAm $(4,0.1,0.01,18) / A A m(4,0.1,0.01,18)$ & PAAm \\
\hline DMA $(4,0.1,0.01,18) / \operatorname{DMA}(4,0.1,0.01,18)$ & PDMA \\
\hline
\end{tabular}

Mechano-Optical Measurements. A real-time mechano-optical measurement platform equipped with a CCD camera was used to study the deformation behavior of the DN hydrogels. Details of the equipment are discussed elsewhere [15]. In short, a rectangular specimen was clamped to clamps attached to two crossheads of the uniaxial stretching machine. Both top and bottom crossheads of this custom-made machine move in opposite directions during stretching, which allows the center of the specimen to remain stationary during extension. The analysis of the mechanical behavior assumed that the material was incompressible and the deformation was isotropic in the non-stretching directions. Thus,

$$
\begin{gathered}
D_{0} W_{0} L_{0}=D_{t} W_{t} L_{t}, \\
\frac{W_{t}}{W_{0}}=\frac{D_{t}}{D_{0}},
\end{gathered}
$$


where $D, W$ and $L$ are the thickness, width and length of the specimen and the subscripts 0 and $t$ denote the initial and instantaneous values, i.e., at time $=0$ and time $=\mathrm{t}$, respectively. The width of the center cross-sectional area was measured in real-time by a laser micrometer, and the true stress, $\sigma_{T}$, engineering strain, $\varepsilon_{e}$, and Hencky (true) strain, $\varepsilon_{H}$, were calculated by

$$
\begin{gathered}
\sigma_{T}=\frac{F_{t}}{W_{t} D_{t}}=\frac{F_{t}}{\left[D_{0}\left(\frac{W_{t}^{2}}{W_{0}}\right)\right]}, \\
\varepsilon_{e}=\frac{L_{t}-L_{0}}{L_{0}}=\left(\frac{W_{0}}{W_{t}}\right)^{2}-1, \\
\varepsilon_{H}=\operatorname{Ln}\left(\frac{L_{t}}{L_{0}}\right)=\operatorname{Ln}\left[\left(\frac{W_{0}}{W_{t}}\right)^{2}\right],
\end{gathered}
$$

where $F_{t}$ is the instantaneous force at time $=t$. A visible wavelength light source $(\lambda=546 \mathrm{~nm})$ was used to measure the optical retardation $(\Gamma)$ at the center of the specimen cross-section. The in-plane birefringence $\left(\Delta n_{12}\right)$ was calculated by

$$
\Delta n_{12}=\frac{\Gamma}{D_{t}}=\frac{\Gamma}{\left(\frac{W_{t} D_{0}}{W_{0}}\right)} .
$$

The DN hydrogel specimens were stretched at room temperature at three different extension rates, 5,50 and $500 \mathrm{~mm} / \mathrm{min}$. Hereafter, the tensile experiments will be represented by the gel notation shown in Table 1, plus the extension rate used in the tensile test. Therefore, PAAm-50 signifies a PAAm DN gel that was extended at a rate of $50 \mathrm{~mm} / \mathrm{min}$ from both sides. The samples were extended to nearly three times of their initial length $\left(\varepsilon_{\mathrm{e}}=2.00 ; \varepsilon_{\mathrm{H}}=1.10\right)$ and 
then held at that strain for $10 \mathrm{~min}$, during which the stress relaxation was monitored. The design of the machine imposed a limitation of $\varepsilon_{\mathrm{H}}=1.10$.

It should be noted that the rate of evaporation of water in the experiments was negligible. Weighing the sample before and after the slowest tensile test indicated that the sample lost less than $2 \%$ of its weight. As will be shown later in this paper, the coincidence of data points for tensile experiments performed at different extension rates is consistent with the negligible evaporation of water during experiments.

Refractive Index Measurements. Refractive indices of PAAm and PDMA hydrogels in both wet and dried states were measured by a Bellingham + Stanley Abbe 60/HR refractometer. The refractive indices were measured against the measuring prism of the Abbe refractometer and an immersion liquid with refractive index of 1.69 was used to improve the contact between the prism and the sample surfaces. A white light source with a band pass filter $(633 \mathrm{~nm})$ was used to generate the monochromatic light. 


\section{RESULTS AND DISCUSSION}

Figure 1 shows the true stress vs. true strain (Hencky) results of tensile tests performed on the DN hydrogels at different extension rates and the stress relaxation results while the stretched sample was held at $\varepsilon_{\mathrm{H}}=1.10$ for $10 \mathrm{~min}$. The stress-strain behavior of the PAAm and PDMA hydrogels was similar to that of an extensible biological tissue [16]. This stress-strain behavior is observed in tensile deformation of most of extensible biological tissues such skin, gut wall, artery, bladder [17]. As will be discussed later in this paper, the similarity to biological tissue is important with regard to choosing an elastic potential for deriving a constitutive equation that describes the mechanical behavior of the DN hydrogels.

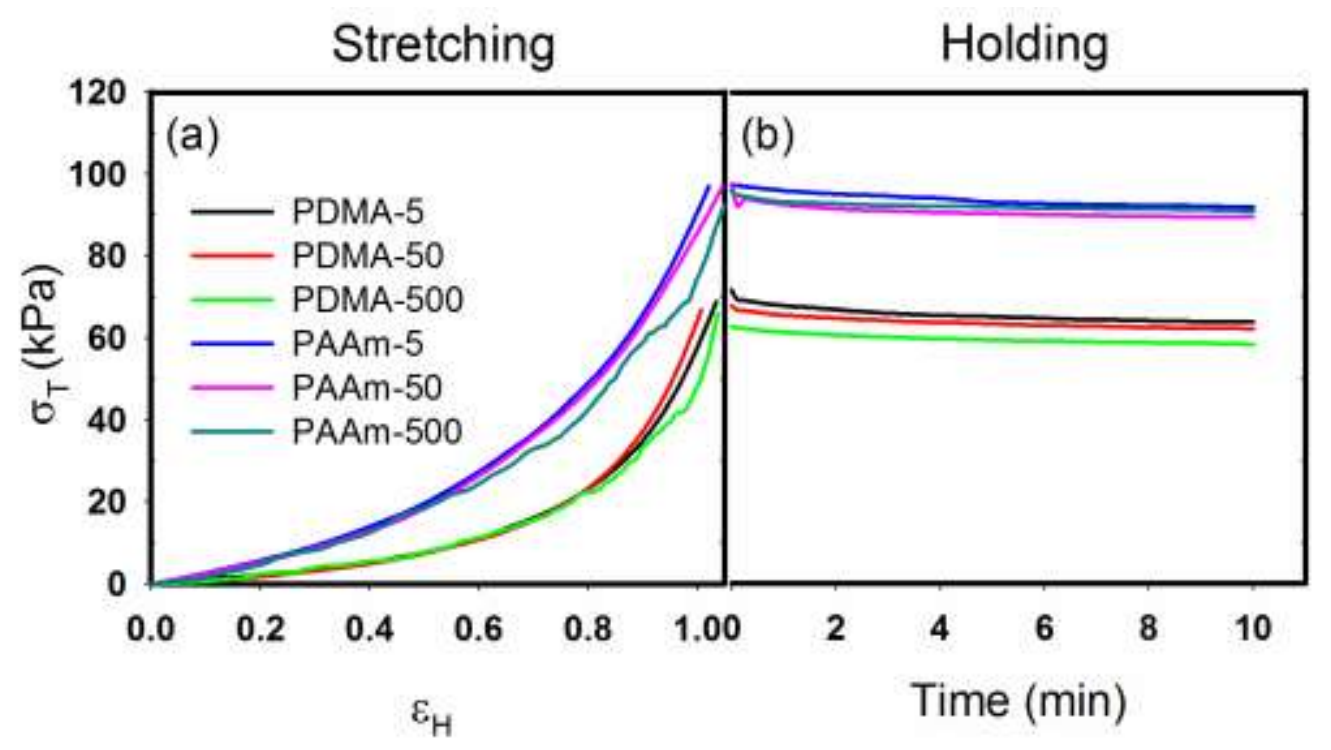

Figure 1 Stretching PAAm and PDMA DN hydrogels at different extension rates followed by 10 min holding. (a): true stress versus true strain, (b): true stress versus time.

A comparison between tensile data performed at different extension rates from quasi-static to a very rapid test indicates that remarkably both hydrogels exhibited rate-independent tensile behavior. The small deviation in the tensile response of the two samples measured at 500 
$\mathrm{mm} / \mathrm{min}$ was due to the acceleration effect. When the rate of extension is very high, acceleration effect can result in stress wave propagation in the sample [18]. In quasi-static experiments the acceleration effect is insignificant and there is no stress wave propagation in the test specimen. Later in this paper when the constitutive equation for the gels is derived, the model will be compared with the experimental data obtained at quasi-static condition $(5 \mathrm{~mm} / \mathrm{min})$.

The results of relaxation after tensile tests are also consistent with the tensile data indicating a rate-independent mechanical behavior. The stress relaxation results indicate that, the stress did not change markedly during holding at the end of each tensile test. A limitation was imposed on the time of relaxation test due to evaporation of water. Although the relaxation time is not very long, the relaxation rate is extremely slow. It is important to note that in viscoelastic solids, the stress usually decreases quite rapidly at first and then gradually approaches a limiting value [19]. In a polymer that has a network structure, such as PAAm and PDMA gels in this work, the orientation imposed by deformation cannot be dissipated by ordinary relaxation processes [20] (See birefringence during relaxation in Figure 2).

Although the same concentration of monomers and initiator was used for polymerization of the PAAm and PDMA gels, PAAm achieved higher stress values as compared to PDMA. This result is attributed to the different reactivity ratios of the monomers AAm and DMA with respect to the crosslinking monomer (MBAA). Bae et al. [21] synthesized PAAm and PDMA hydrogels using the similar ratio of monomer/cross-linker/initiator. They observed similar difference in mechanical behavior of hydrogels in that PAAm gel sustained higher stress values than PDMA hydrogel. They related this behavior to the difference in relative reactivity ratios of the monomers and the crosslinker. In the free radical crosslinking copolymerization the reactivity of crosslinker and the monomer plays a crucial role in topological characteristics of the final 
network [22]. Computer simulations performed by Schröder and Oppermann [23] indicated that the influence of relative reactivity ratios of crosslinking agent and monomer in free radical crosslinking copolymerization decisively affects the properties of the network. For example, the cycle rank which is defined as the number of cuts that can transform a three-dimensional network to a spanning tree and determines the elastic properties of the network is highly influenced by the reactivity ratios [23].

Figure 2 shows the variation of strain optical behavior in the hydrogels during the uniaxial tensile test and holding process. Both hydrogels exhibited negative birefringence.

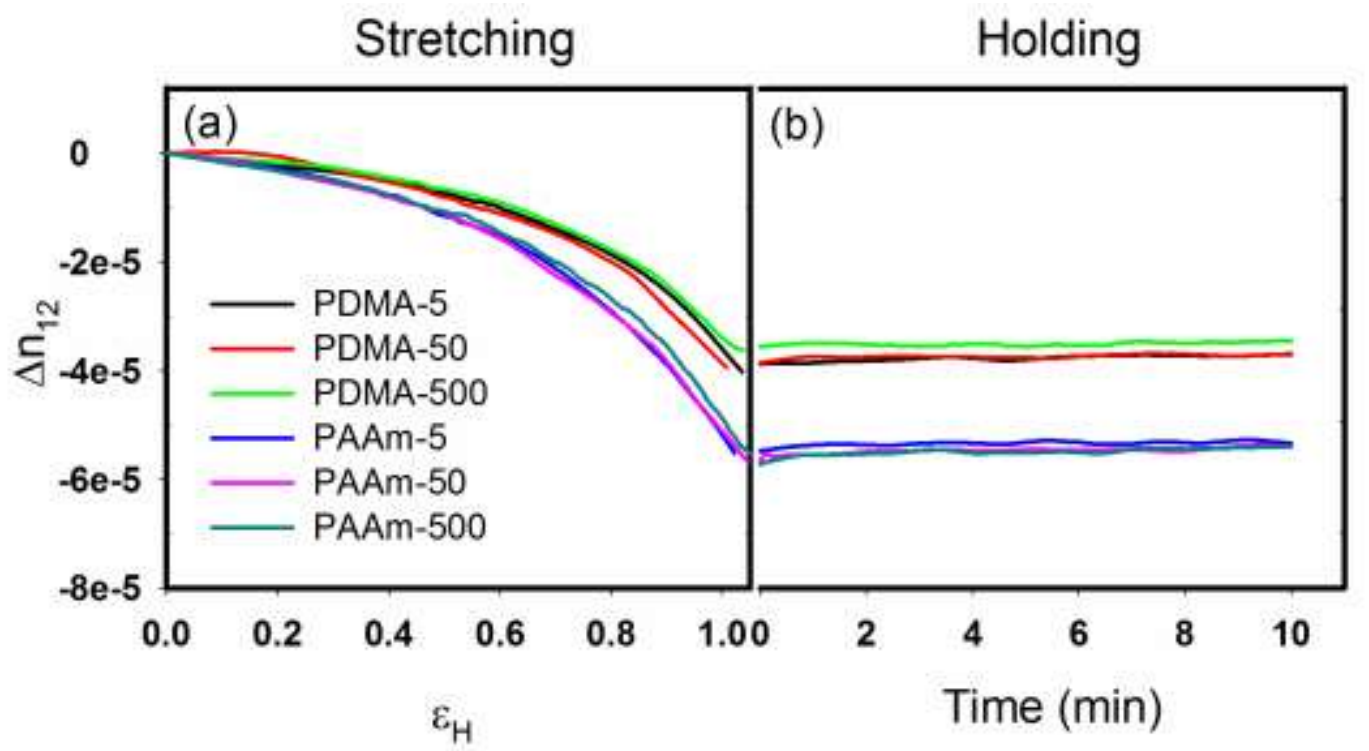

Figure 2 Stretching PAAm and PDMA DN hydrogels at different extension rates followed by 10 min holding. (a): birefringence versus true strain, (b): birefringence versus time.

The absolute value of the birefringence increased with extension and remained constant during the constant strain step after tensile deformation. Negative birefringence for a PAAm hydrogel was also observed by Wu et al. ${ }^{10}$, and Murata and Haraguchi ${ }^{9}$ reported negative birefringence for 
poly( $N$-isopropyl acrylamide) hydrogel. The higher absolute values of the birefringence shown in Figure 2 for the PAAm gels correspond to higher orientation of the network chains, which is consistent with the higher stresses generated by the deformation, as shown in Figure 1.

The magnitude of the birefringence in a tensile experiment depends on the stress optical coefficient of the material. Figure 3 shows the relationship between the birefringence and the true stress for the DN hydrogels for the different stretching rates. As mentioned earlier, the pristine stress optical data can only be obtained in a real-time mechano-optical experiment. The data for the PAAm DN gels in Figure 3 are linear, but the PDMA DN hydrogels exhibited nonlinear birefringence-stress behavior.

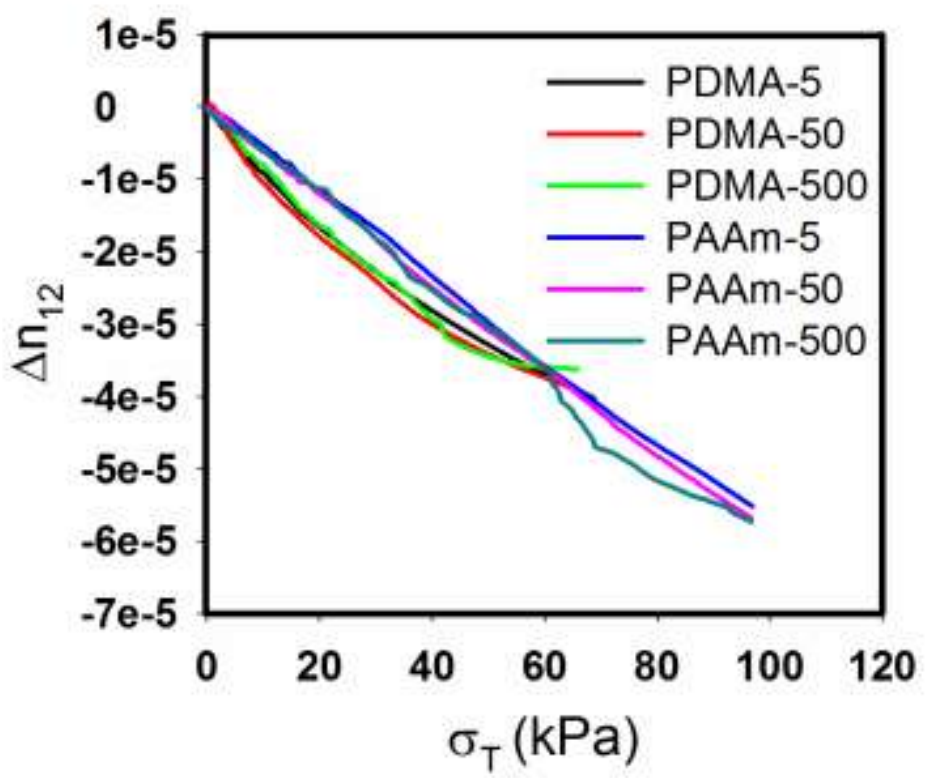

Figure 3 Birefringence versus true stress for PAAm and PDMA DN hydrogels.

The stress-optical law predicts a linear relationship between birefringence and stress, indicating that the optical anisotropy of an isotropic material under an applied stress is 
proportional to the stress and the proportionality constant is called the stress-optical coefficient $[24,25]$. Figure 3 shows for the first time, the variation of birefringence as a function of true stress in finite strain tensile deformation of chemically crosslinked hydrogels. The important observation herein is that stress-optical law holds for PAAm, however, birefringence-true stress deviates from linear behavior for PDMA. It has been confirmed that rubbers obey the stressoptical law even under finite deformations where stress-strain behavior becomes nonlinear $[24,25]$. The observation in Figure 3 raises the question of why PDMA deviates from the stressoptical law. To answer this question, one must assess potential causes for such. For example, any factor that can result in birefringence unrelated to the molecular orientation will give rise to the departure from stress-optical law [26]. A hydrogel can be envisioned as a mixture of a hydrophilic polymer network and a large amount of water. In other words it can be considered as an elastic polymer solution [27]. It is important to note that, in the mixtures of two components like polymer solutions and gels, the difference in dielectric constant of the two components, polymer chains and the solvent, creates an anisotropic internal field that can contribute to the birefringence but its contribution is not accounted for by the stress-optical law [24]. In effect, when the refractive index of the macromolecule is different from that of the solvent, due to the anisotropic internal field, additional anisotropy of the macromolecules arises as a result of optical interaction between separate parts of the chain. Therefore, in addition to intrinsic birefringence, the anisotropy due to the macroscopic shape of polymer chains (macroform anisotropy) and the anisotropy due to the optical interactions of adjacent chain segments (microform anisotropy) also contribute to the birefringence [28]. In the derivation of stress-optical law, only the intrinsic birefringence is taken into account. Thus, macro-and microform anisotropy can be the reason for the breakdown of stress-optical law. It is important to note that both macro- and microform 
anisotropy decrease by increasing the concentration of polymer [28]. In other words, in very dilute systems the macro- and microform anisotropy become more important.

Table 2 shows the water contents, and refractive indices of PAAm and PDMA hydrogels in their wet and dried states.

Table 2. Water contents and refractive indices of the DN Hydrogels

\begin{tabular}{cccc}
\hline Hydrogel & $\begin{array}{c}\text { Water content } \\
(\mathbf{w} \mathbf{\%})\end{array}$ & $\begin{array}{c}\text { Refractive index } \\
\text { (wet gel) }\end{array}$ & $\begin{array}{c}\text { Refractive index } \\
\text { (dry gel) }\end{array}$ \\
\hline PAAm & 92.23 & 1.343 & 1.548 \\
\hline PDMA & 96.30 & 1.338 & 1.558 \\
\hline
\end{tabular}

As one can see, the refractive indices of both hydrogels are different from the refractive index of water that is 1.333 . Therefore, macro-and microform anisotropy contribute to the birefringence. The difference between refractive index of polymer and solvent is higher in case of PDMA and PDMA hydrogel contains more water as compared to PAAm. According to above-mentioned explanation regarding the role of micro- and macroform anisotropy on deviation from stress-optical low and recalling the fact the in more dilute systems this effect becomes more pronounced the nonlinear stress-optical low in PDMA is attributed to the macroand microform anisotropy. The nonlinear birefringence - true stress behavior in PDMA can also be explained by making a comparison between chemical structure of PDMA and PAAm hydrogels, see schemes 1 and 2. As mentioned above, in polymer solutions and hydrogels, the macro-and microform anisotropy originate from the difference in the isotropic part of the polarizability between polymer segments and solvent molecules [24]. The presence of two nonpolar methyl groups in the DMA structure results in higher difference in polarizability 
between the PDMA network and water molecules. This explanation is consistent with the difference between the refractive indices of dry gels and water. Thus, a higher contribution from macro-and microform anisotropy is expected in case of PDAM hydrogel that has been ignored in the derivation of the linear stress-optical law. In the next section, in the development of the photoelastic model it will be shown that estimation of stress-optical coefficient from the linear part of the data at very low stress values for PDMA hydrogel could describe experimental data but a deviation was observed at higher stress values.

\section{THEORETICAL CONSIDERATIONS}

The finite deformation of DN hydrogels is modeled by considering the DN hydrogel as an isotopic, incompressible medium undergoing isothermal rate-independent finite deformation. For the 3-dimensional formulation of isothermal, incompressible finite isotropic elasticity the Cauchy (true) stress tensor $\underline{\underline{\sigma}}$ is given by [29]

$$
\underline{\underline{\sigma}}=-p \underline{\underline{\delta}}+2 W_{1} \underline{\underline{B}}-2 W_{2} \underline{\underline{B}}^{-1}
$$

where $p$ is the pressure, $\underline{\underline{\delta}}$ is the unit tensor, $\underline{\underline{B}}$ is the Finger strain tensor, $W=W\left(I_{1}, I_{2}\right)$ is the strain energy density function, $I_{1}=\operatorname{tr}(\underline{\underline{B}})$ and $I_{2}=\operatorname{tr}\left(\underline{\underline{B}}^{-1}\right)$ are the first two invariants of the Finger strain tensor, and $W_{k}=\partial W / \partial I_{k}$.

For uniaxial extension with stretch ratio, $\lambda$, the deformation gradient tensor is $\underline{\underline{F}}=\operatorname{diag}(\lambda, 1 / \sqrt{\lambda}, 1 / \sqrt{\lambda})$ and the invariants of the Finger strain tensor, $\underline{\underline{B}}=\underline{\underline{F}} \underline{\underline{F}}^{T}$ are

$$
I_{1}=\lambda^{2}+\frac{2}{\lambda}, \quad I_{2}=2 \lambda+\frac{1}{\lambda^{2}}
$$

For uniaxial extension of an incompressible, isotropic material, the Cauchy stress in terms of $\lambda$ is 


$$
\sigma_{11}=2 W_{1}\left(\lambda^{2}-\frac{1}{\lambda}\right)+2 W_{2}\left(\lambda-\frac{1}{\lambda^{2}}\right)
$$

Since the mechanical behavior of the DN hydrogels is similar to that of an extensible biological tissue, the strain energy density function in Eq. (9) was substituted by the Fung elastic potential [16],

$$
W=\frac{\mu}{2 \beta}\left(e^{\beta\left(I_{1}-3\right)}-1\right)
$$

where $\mu$ and $\beta$ are material parameters that can be determined by fitting Eq. (11) for the Cauchy stress,

$$
\sigma_{11}=\mu\left(\lambda^{2}-\frac{1}{\lambda}\right) e^{\beta\left(\lambda^{2}+\frac{2}{\lambda}-3\right)}
$$

After deriving the constitutive equation, the birefringence is expressed by the stress-optical law,

$$
\Delta n_{12}=C \mu\left(\lambda^{2}-\frac{1}{\lambda}\right) e^{\beta\left(\lambda^{2}+\frac{2}{\lambda}-3\right)},
$$

where $C$ is the stress-optical coefficient.

The constitutive equation, Eq. (11), was fit to the experimental tensile data in Figure 1 to obtain $\mu$ and $\beta$, which are summarized for each gel in Table 3. Although the data for different extension rates coincided, the tensile data at the quasi-static condition, $5 \mathrm{~mm} / \mathrm{min}$, was considered for modeling in order to be sure that acceleration effects are minimized. Figure 4 shows the comparison of the model with experimental data, stress vs. stretch ratio, for both DN hydrogels. The number of data points was reduced to make a clearer comparison with model, which is shown by the solid lines. The model described the experimental data well, and the material constants $\mu$ and $\beta$ calculated for each hydrogel are given in Table 3 . 
Table 3. Model parameters and stress-optical coefficients of the hydrogels

\begin{tabular}{cccc}
\hline Hydrogel & $C \times 10^{6}\left(\mathrm{kPa}^{-1}\right)$ & $\mu(\mathrm{kPa})$ & $\beta$ \\
\hline PAAm & -0.586 & 8.78 & 0.0743 \\
\hline PDMA & -0.897 & 2.93 & 0.199 \\
\hline
\end{tabular}

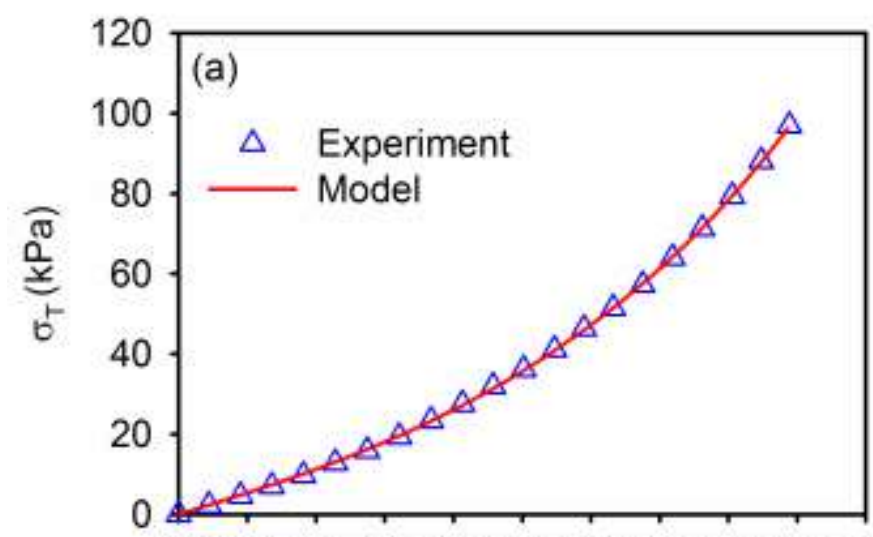

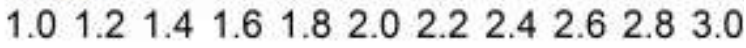

$\lambda$

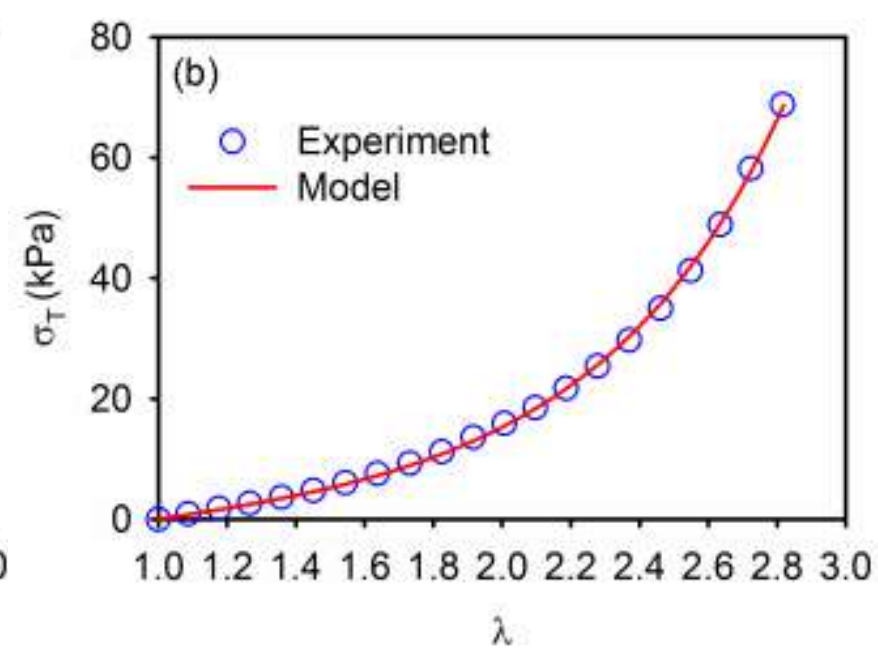

Figure 4 Comparison of experimental data with the model, Eq. (11). Data points represents true stress as a function of stretch ratio and the solid line shows the model. (a): PAAm-5; (b): PDMA-5.

The stress-optical coefficient of the PAAm hydrogel was calculated from the slope of the birefringence-stress data obtained at extension rate of $5 \mathrm{~mm} / \mathrm{min}$. Since the experimental data in Figure 3 for the PDMA hydrogel were not linear, $C$ was estimated by the linear region at very low stress. The stress-optical coefficients for the DN hydrogels are also given in Table 3.

The parameters in Table 3 were used in Eq. (12) to calculate birefringence as a function of stretch ratio, which are compared to the experimental data in Figure 5. Again, the number of data points used was reduced to make a clearer comparison of the data with the model, which is shown by solid lines. Although the model successfully captured the birefringence data of PAAm hydrogel, Figure 5(a), a deviation from experimental data was observed in case of PDMA 
hydrogel, Figure 5(b). The deviation from model for PDMA hydrogel is attributed to the way we estimated the stress-optical coefficient from the nonlinear birefringence - stress curve. Eq. (12) was derived based on the linear stress-optical law. Therefore, using this linear relationship with stress-optical coefficient estimated at very low stress values cannot describe the birefringence data in the whole range of stretch ratios.
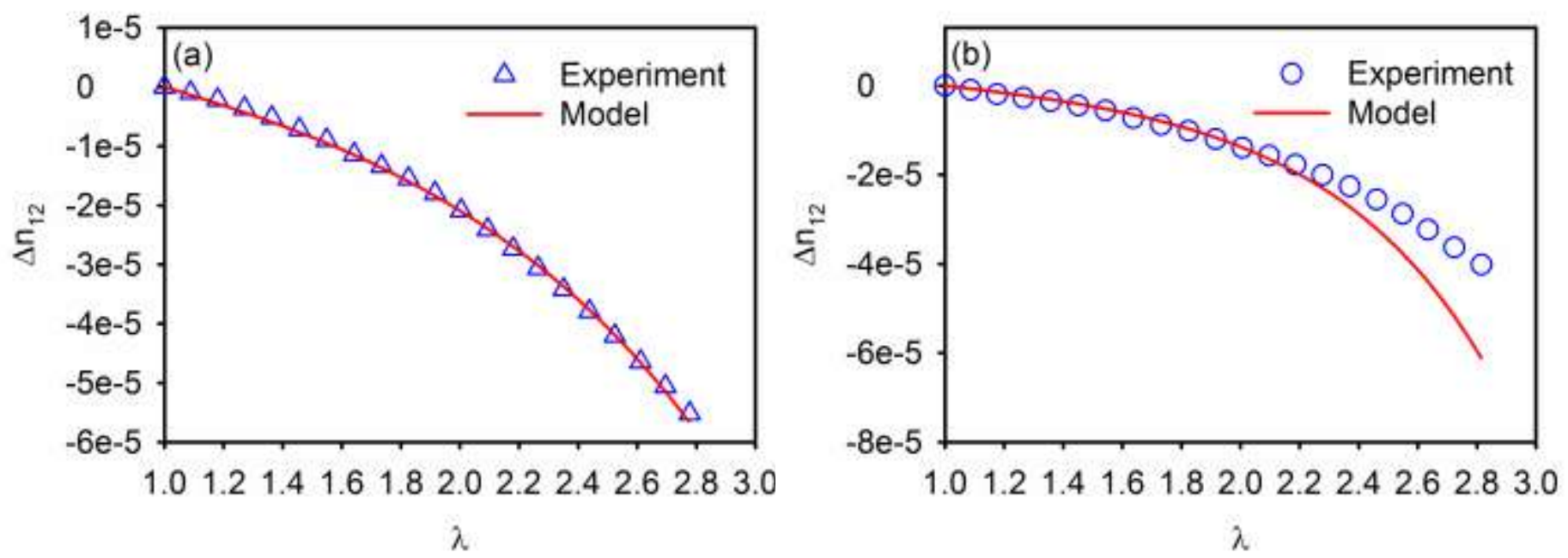

Figure 5 Comparison of experimental data with the model, Eq. (12). Data points represents birefringence as a function of stretch ratio and the solid line shows the model. (a): PAAm-5; (b): PDMA-5. 


\section{CONCLUSIONS}

For the first time photoelasticity of chemically crosslinked hydrogels synthesized from acrylamide (AAm) and N,N-dimethyl(acrylamide) (DMA) was studied by real-time birefringence measurements during finite tensile deformation. One of the important experimental results in this study which is crucial in determining the photoelastic behavior of the material was the birefringence-true stress curve that cannot be obtained readily with off-line measurements.

Free-radical polymerization of a loosely crosslinked polymer network made from AAm or DMA inside the same network can produce a tough double-network (DN) hydrogel whose stress-strain behavior is similar to that of an extensible biological tissue. The chemical structure of these DN hydrogels plays a crucial role in their mechano-optical behavior. Both DN hydrogels exhibit negative birefringence whose absolute value increases with extension and stays constant during relaxation. The stress-optical law holds for PAAm, however, a deviation from linear birefringence - true stress behavior is exhibited by PDMA. The departure from stress-optical law in PDMA is attributed to the contribution of macro- and microform anisotropy to the birefringence that originates from the difference in refractive indices of polymer and the solvent.

A constitutive equation based on Fung elastic potential can perfectly describe the tensile behavior of the gels. The stress-optical coefficients of hydrogels can be found from the birefringence - stress curve. In case of a nonlinear birefringence - stress behavior, stress-optical coefficient may be estimated by the slope of the curve at very low stress values. In such a case, the constitutive equation derived based on the Brewster's stress-optical law cannot describe the experimental data in the whole range of extension and a deviation from experimental data is expected at extensions corresponding to the high values of stress. 


\section{ACKNOWLEDGMENT}

This research was supported in part by a grant from the Civil, Mechanical and Manufacturing Innovation (CMMI) Division within the Engineering Directorate of the National Science Foundation, CMI-1300212.

\section{NOTATION}

$\underline{\underline{\sigma}} \quad$ Cauchy stress tensor

$p \quad$ pressure

$\underline{\delta} \quad$ unit tensor

$W \quad$ strain energy density tensor

$\underline{B} \quad$ Finger strain tensor

$\underline{B}^{-1} \quad$ inverse of Finger strain tensor

$\operatorname{tr}(\underline{\underline{B}}) \quad$ trace of Finger strain tensor

$I_{1}, I_{2} \quad$ first and second invariants of Finger strain tensor

$\lambda \quad$ stretch ratio

$\underline{F} \quad$ deformation gradient tensor

diag diagonal matrix

$\mu, \beta \quad$ material parameters

$\Delta n_{12} \quad$ birefringence

$C \quad$ stress-optical coefficient

$\varepsilon_{H} \quad$ true strain 


\section{REFERENCES}

[1] H. F. Mark, Encyclopedia of Polymer Science and Technology, Vol. 2; John Wiley \& Sons, Inc.: New York, 2007.

[2] R. M. Ottenbrite, K. Park, T. Okano, Biomedical Applications of Hydrogels Handbook, Springer Science+Business Media, LLC 2010.

[3] S. Kirchhof, A. M. Goepferich, F. P. Brandl, Hydrogels in Ophthalmic Applications, European Journal of Pharmaceutics and Biopharmaceutics 95 (2015) 227-238.

[4] W. Fiala, Birefringent Multifocal Lenses: Theory and Application to the Correction of Refractive Error, Optometry \& Vision Science 67 (1990) 787-791.

[5] A. Amigó-Melchior, H. Finkelmann, A Concept for Bifocal Contact-or Intraocular Lenses: Liquid Single Crystal Hydrogels (“LSCH”), Polym. Adv. Technol. 13 (2002) 363-369.

[6] J. Hrouz, M. Ilavský, Phase Transition in Swollen Gels, Polym. Bull. 12 (1984) 515-521.

[7] E. Geissler, A. M. Hecht, J. Torbet, Spontaneous Birefringence and Photoelasticity of Polyacrylamide Gels, Polymer 27 (1986) 1489-1492.

[8] X. Zhao, Multi-scale Multi-Mechanism Design of Tough Hydrogels: Building Dissipation into Stretchy Networks, Soft Matter 10 (2014) 672-687.

[9] K. Murata, K. Haraguchi, Optical Anisotropy in Polymer-Clay Nanocomposite Hydrogel and Its Change on Uniaxial Deformation. J. Mater. Chem. 17 (2007) 3385-3388.

[10] Z. L. Wu, D. Sawada, T. Kurokawa, A. Kakugo, W. Yang, H. Furukawa, J. P. Gong, Strain-Induced Molecular Reorientation and Birefringence Reversion of a Robust, Anisotropic Double-Network Hydrogel, Macromolecules 44 (2011) 3542-3547.

[11] J. P. Gong, Y. Katsuyama, T. Kurokawa, Y. Osada, Double-Network Hydrogels with Extremely High Mechanical Strength. Adv. Mater. 15 (2003) 1155-1158.

[12] K. Shikinaka, Y. Koizumi, K. Shigehara, Mechanical/Optical Behaviors of Imogolite Hydrogels Depending on Their Compositions and Oriented Structures, J. Appl. Polym. Sci. 132 (2015) $41691(1-6)$.

[13] S. Shams Es-haghi, R. A. Weiss, Fabrication of Tough Hydrogels from Chemically CrossLinked Multiple Neutral Networks, Macromolecules 49 (2016) 8980-8987.

[14] L. R. G. Treloar, The Physics of Rubber Elasticity, Oxford University Press 1975. 
[15] T. L. Kwa, Novel On-Line True Stress-Strain-Electrical Conductivity Uniaxial Tensile Stretching System and Its Utility on Electrically Conductive Polactic Acid (PLA) Nanocomposites. MS Thesis, The University of Akron, May 2006.

[16] Y. C. Fung, Biomechanics, Mechanical Properties of Living Tissues. Springer Science+Business Media New York 1981.

[17] A. G. Atkins, Y. W. Mai, Elastic and Plastic Fracture: Metals, Polymers, Ceramics, Composites, Biological materials. Ellis Horwood, 1985.

[18] J. R. Davis, Tensile Testing, 2nd Edition, ASM International, Materials Park, OH, 2004.

[19] A. C. Pipkin, Lectures on Viscoelasticity Theory, Second Edition, Springer Science+Business Media, LLC 1986.

[20] P. J. Flory, Thermodynamics of Crystallization in High Polymers. I. Crystallization Induced by Stretching. J. Chem. Phys. 15 (1947) 397-408.

[21] Y. H. Bae, T. Okano, S. W. Kim, Temperature Dependence of Swelling of Crosslinked Poly ( $N, N^{\prime}$-alkyl substituted acrylamides) in Water. J. Polym. Sci. Part B: Polym. Phys. 28 (1990) 923-936.

[22] B. Lindemann, U. P. Schröder, W. Oppermann, Influence of The Cross-Linker Reactivity on The Formation of Inhomogeneities in Hydrogels, Macromolecules 30, (1997) 4073-4077.

[23] U. P. Schröder, W. Oppermann, Computer Simulation of Network Formation via Crosslinking Copolymerization. Macromol. Theory Simul. 6 (1997) 151-160.

[24] M. Doi, S. F. Edwards, The Theory of Polymer Dynamics. Oxford University Press Inc. New York 1968.

[25] M. Doi, Introduction to Polymer Physics, Oxford University Press, New York 1996.

[26] C. M. Roland, Viscoelastic Behavior of Rubbery Materials. Oxford University Press. New York 2011.

[27] M. Doi, Soft Matter Physics, Oxford University Press, 2013.

[28] V. N. Tsvetkov, A. E. Grishchenko, Stress Birefringence and Optical Anisotropy of Macromolecules in Swollen Polymers. J. Polym. Sci. Part C: Polym. Symp. 16 (1967) 31953204.

[29] C. Truesdell, W. Noll, The Non-Linear Field Theories of Mechanics. Springer-Verlag Berlin Heidelberg 2004. 


\section{Graphical Abstract}

Mechano-Optical Behavior of Loosely Crosslinked Double-Network Hydrogels: Modeling and Real-Time Birefringence Measurement during Uniaxial Extension

S. Shams Es-haghi, ${ }^{1,2,3}$ I. Offenbach, ${ }^{1 \dagger}$ D. Debnath, ${ }^{1}$ R. A. Weiss, ${ }^{1}$ M. Cakmak ${ }^{1,2,3,4^{*}}$

${ }^{1}$ Department of Polymer Engineering, The University of Akron, Akron, Ohio, 44325-0301, USA

${ }^{2}$ Birck Nanotechnology Center, Purdue University,

West Lafayette, Indiana 47907-2057, USA

${ }^{3}$ School of Materials Engineering, Purdue University,

West Lafayette, Indiana 47907-2045, USA

${ }^{4}$ School of Mechanical Engineering, Purdue University,

West Lafayette, Indiana 47907-2088, USA
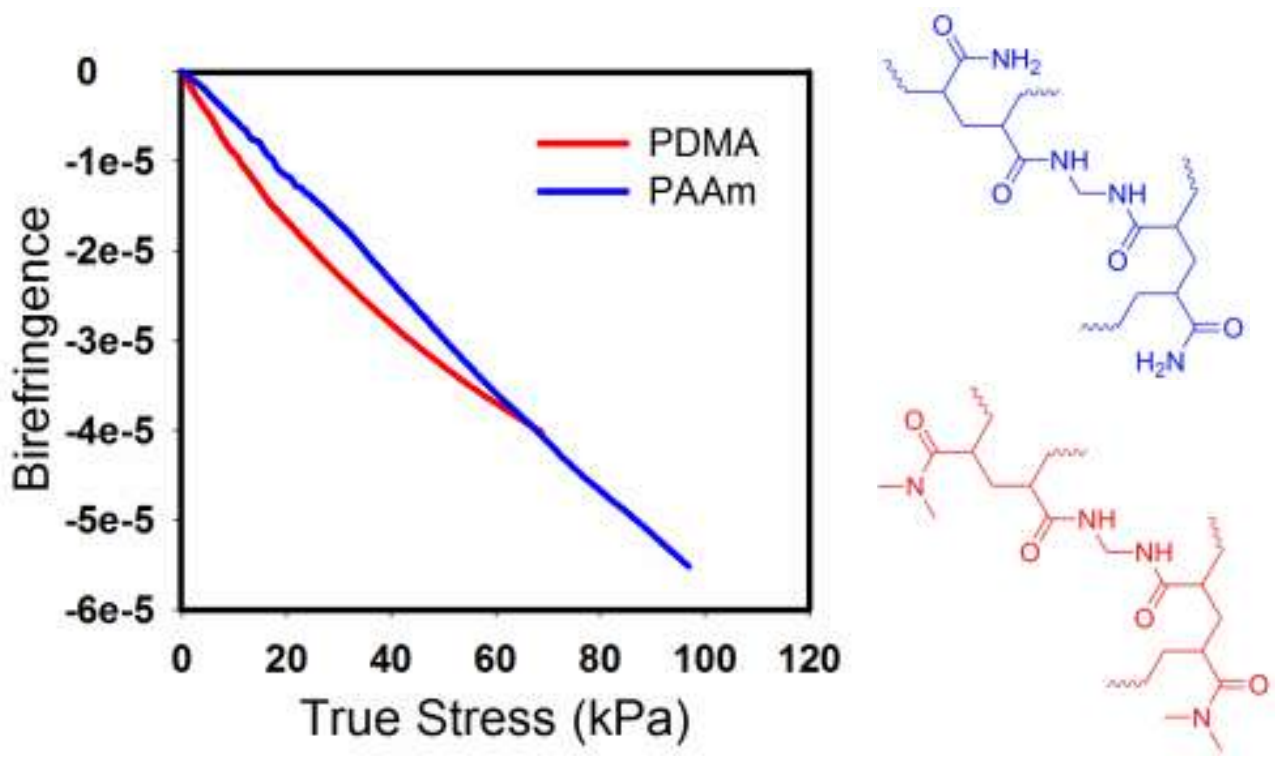
*Corresponding author: cakmak@purdue.edu

${ }^{\dagger}$ Current address: Evonik Corporation, Nutrition care Division, Richmond, Virginia 23237, USA 